\title{
Nonlinear pulses at the interface and its relation to state and temperature
}

\author{
Kevin H. Kang a and Matthias F. Schneider ${ }^{\mathrm{b}}$ \\ Department of Physics, Technical University of Dortmund, Dortmund, Germany \\ Received 7 June 2019 and Received in final form 13 January 2020 \\ Published online: 5 February 2020 \\ (C) 2020. This article is published with open access at Springerlink.com
}

\begin{abstract}
Environmental temperature has a well-conserved effect on the pulse velocity and excitability of excitable biological systems. The consistency suggests that the cause originates from a fundamental principle. A physical (hydrodynamic) approach has proposed that the thermodynamic state of the hydrated interface (e.g., plasma membrane) determines the pulse behavior. This implies that the temperature effect happens because the environmental temperature affects the state of the interface in any given system. To test the hypothesis, we measured temperature-dependent phase diagrams of a lipid monolayer and studied the properties of nonlinear acoustic pulses excited along the membrane. We observed that the membrane in the fluid-gel transition regime exhibited lower compressibility (i.e., stiffer) overall with increasing temperature. Nonlinear pulses excited near the transition state propagated with greater velocity with increasing temperature, and these observations were consistent with the compressibility profiles. Excitability was suppressed significantly or ceased completely when the state departed too far from the transition regime either by cooling or by heating. The overall correlation between the pulses in the membrane and in living systems as a function of temperature supports the view that the thermodynamic state of the interface and phase transition are the key to understanding pulse propagation in excitable systems.
\end{abstract}

\section{Introduction}

Environmental temperature has a conserved effect on pulse propagation in diverse biological systems (hereafter referred to as "temperature-conduction relation"). In particular, increased temperature increases conduction velocity in squid axon fibers, frog motor nerve, cat axon, rabbit Purkinje fiber, rat cardiomyocytes, sea slug neurons, algae, worm blood vessels, fish spinal cord, etc. [1-10]. In addition, pulse duration decreases, and pulse frequency (rate) accordingly increases with temperature. However, excitability is lost below and above a certain temperature range (commonly known as cold/heat-block), and the conduction velocity reaches a maximum slightly below the heat-block temperature. The overall consistency of these observations across species and cell types leaves no doubt that the temperature-conduction relation is not a coincidence, but a consequence of a fundamental principle. Here we investigate to identify the origin of this rather remarkable phenomenon.

\footnotetext{
* Supplementary material in the form of a .tif file available from the Journal web page at

We address the question using classical thermodynamics. This is partly because all phenomena, including quantum and biological, must follow the laws of thermodynamics and accordingly be explainable in the language of thermodynamics. It also has broad applicability. Applying thermodynamics has a limitation in obtaining exact understanding of the behavior of complex systems because one often neglects the underlying nature of the system. Because of this, however, the findings deduced are not restricted to a particular system. Finally, thermodynamics directly involves temperature, a macroscopic quantity that can be accessed and controlled. This approach therefore is relevant for understanding a common physiological behavior that arises from diverse systems such as the temperature dependence. Ongoing evidences have advocated that pulse conduction is a macroscopic phenomenon that directly involves macroscopic structures such as the membrane and/or cytoskeleton $[11-13]$. We have hypothesized that the pulse propagation depends on the thermodynamic state of the interface of excitable biological system (e.g., plasma membrane), and we observed supporting evidences particularly concerning velocity, excitability, and nonlinearity [14-16]. Regarding the temperature-conduction relation, the hypothesis leads to the prediction that the relation is observed because temperature affects the state of the interface. This arises rather trivially because if the membrane is a com- 
pletely defined, thermally open system, its state is altered by the temperature-dependent heat exchange with its surrounding (i.e., environment) [17]. However, whether pulse conduction indeed follows directly from the state changes due to temperature has not been determined.

Here we investigated whether a state-behavior relation exist to determine whether the temperature relation originates from the interface. We test this by studying the propagation of nonlinear acoustic pulses on a model membrane at different temperatures. Briefly, state diagrams of the monolayer were measured within a temperature range. Pulses were generated by local compression of the membrane near the phase transition, and the characteristics of the waves, such as speed, amplitude, and excitability, were analyzed with respect to the state of the interface. We then compared our observations to those observed in living systems. Because mechanical and electrical pulses are coupled thermodynamically [18-20], we deemed that studying acoustic pulses is a suitable method to test the hypothesis. Regarding the state, additional attention was placed on the compressibility of the membrane not only because it is relevant in acoustics, but also our previous work suggested that the temperature dependence of pulse propagation and excitation are predictable by the temperature dependence of the compressibility [9].

\section{Materials and methods}

Preparation and excitation of monolayer were performed in similar fashion as described previously [15, 21]. Briefly, 1,2-dipalmitoyl-sn-glycero-3-phosphocholine (DPPC) was mixed with FRET-pair fluorophores at 98:1:1 w/v\% (lipid: DPPC, Avanti Polar; Alabaster, AL, USA; donor/acceptor dye: NBD-PE/Texas Red DHPE, Thermo Fischer Scientific; Waltham, MA, USA). The solution was then spread on a Langmuir film balance (NIMA; Coventry, England) on the air-water (18 M $\Omega$ ) interface. Using a heat exchanger (VWR International; Pennsylvania, PA, USA) underneath the trough, the subphase temperature was regulated to within $\pm 0.5^{\circ} \mathrm{C}$ of the target temperature. FRET emission was coupled to the pressure-area state of the monolayer, and its intensity was measured at 540/605 $\pm 12 \mathrm{~nm}$ using a custom epifluorescence microscope fixed at a single position. Acoustic source was generated by perturbing the membrane rapidly with a cantilever embedded on the monolayer (Physik Instrument; Karlsruhe, Germany). The generated pulse was detected $5 \mathrm{~mm}$ from the source, and it was measured based on the relative changes in the intensity from the undisturbed state exhibiting mean density $\rho_{0}$ and intensity $I_{0}$ such that

$$
s=\frac{I-I_{0}}{I_{0}} \sim \frac{\rho-\rho_{0}}{\rho_{0}} .
$$

\section{Results and discussion}

Lateral pressure-area $(\pi-A)$ isotherms were first measured at different temperatures with room temperature $\left(20^{\circ} \mathrm{C}\right)$
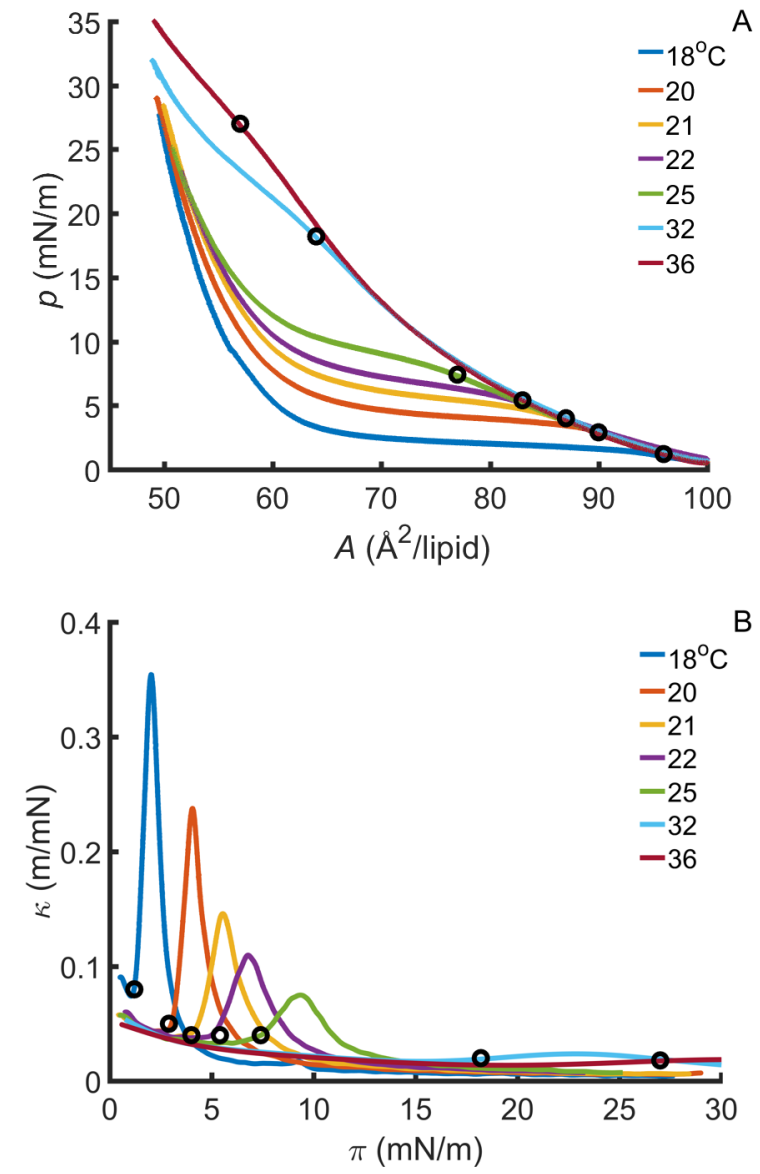

Fig. 1. Thermal phenomenology of the lipid interface. (A) Isothermal pressure-area state diagrams of DPPC monolayer on water with varying subphase temperature. Thermal equilibrium occurred 20-30 minutes after changing the reservoir temperature. Circles indicate the states where membrane was perturbed. (B) Corresponding lateral compressibility $\kappa_{T}$ profiles calculated from the measured isotherms in (A) using eq. (1).

as the reference point. Isotherms indicated that increased temperature to $36^{\circ} \mathrm{C}$ shifts the fluid (liquid-expanded; $\mathrm{LE})$ to the gel (liquid-condensed; LC) transition regime towards higher pressure (fig. 1(A)). The transition regime, indicated by the width of the plateau, also became narrower and steeper, indicating that the first-order transition weakened as the monolayer approached the critical point $\left(\sim 41^{\circ} \mathrm{C}\right)[22]$. State diagrams can also reveal the properties of the system, and in particular, the lateral, isothermal compressibility can be readily accessed from the $\pi-A$ isotherm using eq. (1) [23]:

$$
\kappa_{T}=\frac{1}{A}\left(\frac{\partial A}{\partial \pi}\right)_{T} .
$$

At each temperature, $\kappa_{T}$ exhibited a maximum in the transition regime, and the overall compressibility in the transition regime decreases with increasing temperature. $\kappa_{T}$ in both the LE and LC phase (fluid-gel) were overall not significantly different across temperature. Thus, 
increasing temperature significantly lowers the overall stiffness only in the transition regime, and this finding was consistent with other findings across a broad range of phospholipid species and not exclusive to DPPC [22, $24,25]$.

The relation between temperature and nonlinear sound pulses was then investigated. As demonstrated previously, when the membrane set slightly outside the transition state is perturbed locally with sufficient power, a finite, nonlinear change in the local density relative to the undisturbed state is generated, and this disturbance propagates as a solitary pulse $[15,21]$. Because excitable systems typically exhibit nonlinear behavior (i.e., all-or-none), we studied nonlinear pulses here to compare the pulse characteristics in parallel. To observe this nonlinearity at all temperature, the membrane was set just outside the LELC phase transition for each temperature (fig. 1(A), circles), and it was excited using equal source strength at a fixed distance from the detector. Starting from room temperature $\left(20^{\circ} \mathrm{C}\right)$, the pulse velocity $c(\sim 0.25 \mathrm{~m} / \mathrm{s}[26])$, determined based on when amplitude is at maximum, increased with increasing temperature up to $32^{\circ} \mathrm{C}$ ( $c$ normalized to 1 at room temperature) (fig. 2(A), (B)). From $20^{\circ} \mathrm{C}$, velocity increased by up to 1.62 , which was similar in orders of magnitude in several living systems [1, $3]$. Reducing the temperature down to $18^{\circ} \mathrm{C}$, on the other hand, lowered $c$. Taken altogether, increasing temperature increased the conduction velocity within a range.

To determine whether the velocity measurements can be explained based on the states, we related the measured pulses to the state diagrams. Sound speed $c$ relates to the inverse of the square root of the compressibility $\left(c \sim \sqrt{\kappa_{s}^{-1}}\right)$, and assuming $\kappa_{s} \sim \kappa_{T}[27,28]$, we expected an approximate relation $c \sim \sqrt{\kappa_{T}^{-1}}$. In addition, pulses exhibit nonlinearity when the membrane is compressed into the phase transition region, which leads to a finite condensation $s=\left(\rho-\rho_{0}\right) / \rho_{0}$. Thus, compressibility cannot be treated to be a constant as the pulse propagates, and sound speed becomes a function of condensationdependent compressibility such that $c \sim \sqrt{\kappa(s)_{T}^{-1}}[12$, $26]$. Because the compressibility within the phase transition regime were significantly different across temperature (fig. 1(B)), pulses were expected to undergo different amount of condensation. The exact pulse behavior was not readily predictable, but the general expectation was that pulses at higher temperature would experience a stiffer material as it undergoes a phase transition. Thus, pulse velocity should have increased with temperature, which was indeed observed. Additionally, the amplitude decreased progressively with increasing velocity, indicating that pulses underwent smaller condensation, and this behavior was likewise consistent from the decrease in compressibility. When linear pulses were excited using weaker excitation at the same state, the velocity increased generally (see S.-fig. 1 in the Electronic Supplementary Material (ESM)) due to the overall decrease in the compressibility outside the transition regime (circles, fig. 1(A)). Unlike the nonlinear pulses, however, both the velocity and the

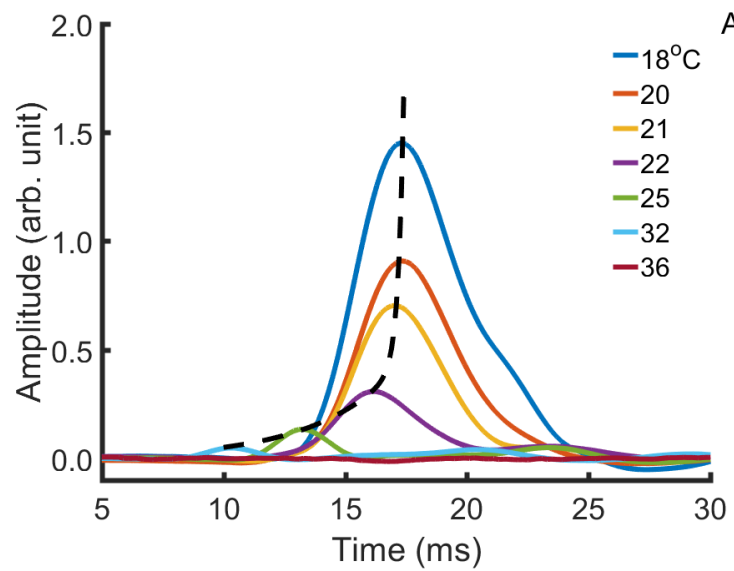

A

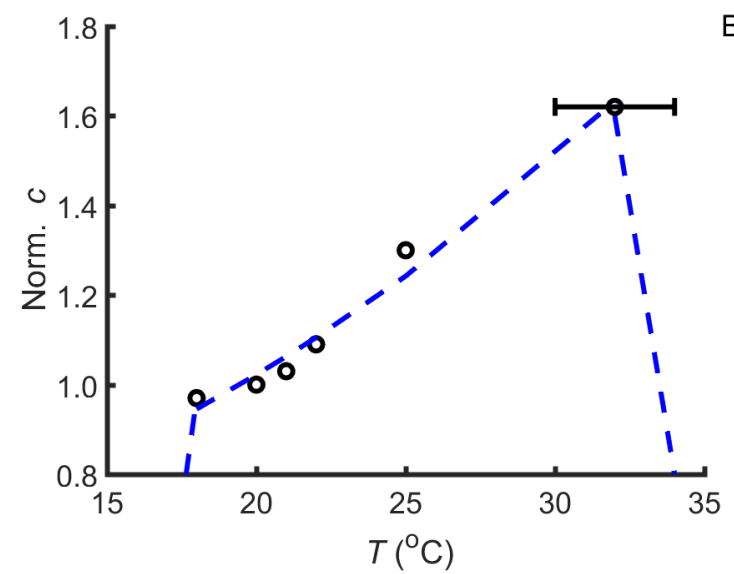

Fig. 2. Variation of pulse propagation along the interface as a function of environmental temperature. (A) Pulse detected at different temperature ( $t=0$ is when pulse was triggered). (B) Comparison of relative changes in speed. Pulse speed is normalized to that at lowest temperature $\left(18^{\circ} \mathrm{C}\right)$. Dotted lines are guides for the eye. Pulses beyond the temperature range (below $18{ }^{\circ} \mathrm{C}$ and above $32^{\circ} \mathrm{C}$ ) were not detected $(n=3-4$, overall amplitude uncertainty $= \pm 5 \%$ ). Pulse speed $c$ is normalized to the room temperature $\left(20^{\circ} \mathrm{C}\right)$.

compressibility did not clearly change monotonically, and amplitude did not change significantly. Because such variations were not observed in nonlinear pulses, the result indicated that pulse behavior was indeed based on the phase transition. Meanwhile, increasing temperature decreases the viscosity of the bulk water, but while such viscous effects are expected to affect pulse transmission by coupling to the membrane [28], it did not explain the decrease in amplitude. Thus, the increase in temperature reduces the overall effective compressibility that the pulse experiences, and the velocity increase is based on the state because it is determined by the thermodynamic property of the membrane.

In addition to conduction velocity, state-dependent excitability was investigated. Increasing the temperature up to $32{ }^{\circ} \mathrm{C}$ increased the pulse speed, but when the temperature was incremented further, pulses were no longer detected (fig. 2(B)). Conversely, when the temperature was 
decreased further below $18{ }^{\circ} \mathrm{C}$, pulses were not clearly resolved as well. Relating these observation to the state diagrams (fig. 1(A)), the absence of pulse coincided with the disappearing of the fluid-gel transition regime towards low and high temperature. This indicated that the cutoff arises because the system loses its nonlinearity. Taking from the results thus far, we further deduce whether this observation can explain the cold and heat block. Even though biological systems typically function under a fixed pressure, we have adjusted the state and likewise the pressure in order to test for nonlinearity. Thus, we consider the case where the system would have been fixed at a particular pressure instead. Supposing that a system is initially excited just outside the transition regime at $22^{\circ} \mathrm{C}$ as the reference point (fig. 1(A), circle), cooling the system while maintaining the pressure would shift the state further towards the transition regime. As this regime is softer, excitation would produce a higher amplitude, but slower signal similar to those observed at lower temperature in fig. 1(B). Further cooling would take the system out of the transition and into the condensed state, where nonlinear compression would no longer occur due to the incapacity to undergo liquid-gel transition. If the temperature is raised on the other hand, fixing the pressure continues to place the system further away from the transition as well. The overall compressibility decreases and pulse velocity increases, but when the temperature is raised sufficiently towards the critical state where the transition starts to vanish, the system is too far from the transition to be excited nonlinearity.

To summarize, we questioned why temperature has a conserved effect in excitable systems, and we hypothesized that this is based on the state of the interface. We excited pulses on a model membrane at different temperatures, and we established that nonlinear pulse velocity increases with increasing temperature. In addition, nonlinear conduction was discontinuous outside a range of temperature. The overall consistency between observations from the membrane and from excitable systems therefore supports the view that the pulse propagation is based on the state of the interface.

In the context of temperature dependence, we discuss briefly the ongoing soliton theory proposed by Heimburg and Jackson in 2005 [12]. The model predicts that conduction velocity depends on the compressibility as well as on the total energy of the soliton. However, it is not clear to us how these two dependences relate to the changes in temperature. Meanwhile, we strongly believe that viscous effects cannot be neglected due to boundary conditions (e.g., evanescent fields), and thus it would be of interest to see how viscous effects (in plane and/or adjacent bulk) would modify the predictions for the velocity in the soliton (or, rather solitary) model.

We detailed evidence relating the general changes in velocity and excitability to the state, but it remains unknown whether this fully explains the relative differences in temperature dependence among excitable systems. Because temperature-velocity variation as well as blocking temperature naturally vary among systems, our study would suggest that the variation between systems arises from the differences in the compressibility. However, the conduction velocity of biological systems ranges several orders of magnitude $(\mathrm{mm} / \mathrm{s}$ to $100 \mathrm{~m} / \mathrm{s})$ [29], and if conduction speed is indeed based entirely on the square root of the compressibility, taking the inverse yields a compressibility range that is unphysical. Even within a system, velocity can vary nearly two orders of magnitude within a $\sim 30^{\circ} \mathrm{C}$ temperature range [7]. Thus, compressibility alone cannot explain the differences, and additional factors must be involved. As such, we expect that the viscous coupling between the interface and its surrounding may explain the differences among various systems, and further studies would support or refute this notion.

As we close, one may ask whether the conduction relation based on other environmental factors besides temperature likewise can be determined by the state. In any excitable system, pulse propagation is influenced not only by the environmental temperature, but also by the type and concentration of the surrounding molecules. For instance, increasing concentration of extracellular $\mathrm{H}^{+}$can decrease conduction velocity, and $p \mathrm{H}$ outside a certain range blocks conduction in a similar manner to the temperature block [30]. Such molecular dependence are often considered to arise exclusively from the interaction between the molecules and specific components of the membrane [31]. However, if the membrane is a thermodynamic ensemble, open or closed, the ubiquitous effect on the entire system should be considered to determine whether the interaction is sufficiently negligible. Based on molecular evidences related to charge screening, protonation, or diffusion into the membrane, all processes involving molecules would unavoidably alter the state of the membrane and hence its fluidity. Such state changes in model membranes clearly occur due to protons [22,32], monovalent and divalent cations [33-36], anesthetics [37,38], and neurotransmitters in the environment [39-41]. Thus, we expect that similarly to the temperature-conduction relation, the molecule-conduction relation is also state dependent, and studying pulse propagation in parallel with phase diagrams of both model membranes and in living systems should further elucidate this matter.

Open Access funding provided by Projekt DEAL. The authors thank Konrad Kaufmann for his ongoing discussions and insights regarding biological communication. The authors also thank Christian Fillafer, Matan Mussel, and Shamit Shrivastava for discussions, and they thank Thomas Rose and Gregor Haider for the supporting experiments.

\section{Author contribution statement}

KHK and MFS designed the experiments. KHK performed the experiments and collected the data. KHK and MFS analyzed the data. KHK and MFS wrote the manuscript.

Publisher's Note The EPJ Publishers remain neutral with regard to jurisdictional claims in published maps and institutional affiliations. 
Open Access This is an open access article distributed under the terms of the Creative Commons Attribution License (http://creativecommons.org/licenses/by/4.0), which permits unrestricted use, distribution, and reproduction in any medium, provided the original work is properly cited.

\section{References}

1. M. Margulis, C.-M. Tang, J. Neurophysiol. 79, 2809 (1998).

2. J.J.C. Rosenthal, F. Bezanilla, J. Exp. Biol. 205, 1819 (2002).

3. A.L. Hodgkin, B. Katz, J. Physiol. 109, 240 (1949).

4. M. Roche, C. Renauleaud, V. Ballet, M. Doubovetzky, J.M. Guillon, J. Pharmacol. Toxicol. Methods 61, 238 (2010).

5. T. Uchida, R. Kitora, K. Gohara, Trends Med. 18, 145 (2018).

6. N.G. Hyun, K.H. Hyun, K. Lee, B.K. Kaang, Neurosignals 20, $252(2012)$.

7. M.J. Beilby, H.G.L. Coster, Funct. Plant Biol. 3, 275 (1976).

8. D. Papahadjopoulos, M. Cowden, H. Kimelberg, Biochim. Biophys. Acta: Biomembr. 330, 8 (1973).

9. C. Fillafer, M.F. Schneider, PLoS One 8, e66773 (2013).

10. B.I. Roots, C.L. Prosser, J. Exp. Biol. 39, 617 (1962).

11. I. Tasaki, Physiology and Electrochemistry of Nerve Fibers (Academic Press, New York, 1982).

12. T. Heimburg, A.D. Jackson, Proc. Natl. Acad. Sci. U.S.A. 102, 9790 (2005).

13. I. Tasaki, Ferroelectrics 220, 305 (1999).

14. J. Griesbauer, S. Bössinger, A. Wixforth, M.F. Schneider, Phys. Rev. E 86, 061909 (2012).

15. S. Shrivastava, M.F. Schneider, J. R. Soc. Interface 11 , 20140098 (2014).

16. B. Fichtl, I. Silman, M.F. Schneider, Langmuir 34, 4914 (2018).

17. L.D. Mosgaard, A.D. Jackson, T. Heimburg, J. Chem. Phys. 139, 125101 (2013)

18. K. Kaufmann, Action Potentials and Electrochemical Coupling in the Macroscopic Chiral Phospholipid Membrane (Caruaru, Brazil, 1989).

19. I. Tasaki, K. Iwasa, R.C. Gibbons, Jpn. J. Physiol. 30, 897 (1980).
20. I. Tasaki, Jpn. J. Physiol. 49, 125 (1999).

21. S. Shrivastava, K.H. Kang, M.F. Schneider, J. R. Soc. Interface 15, 20170803 (2018).

22. O. Albrecht, H. Gruler, E. Sackmann, J. Phys. 39, 301 (1978).

23. L.D. Landau, E.M. Lifshitz, Course of Theoretical Physics, Vol. 7: Theory of Elasticity (Pergamon Press, Oxford, 1970).

24. D. Steppich, J. Griesbauer, T. Frommelt, W. Appelt, A. Wixforth, M.F. Schneider, Phys. Rev. E 81, 061123 (2010).

25. T. Heimburg, Thermal Biophysics of Membranes (John Wiley \& Sons, 2008).

26. S. Shrivastava, K.H. Kang, M.F. Schneider, Phys. Rev. E 91, 012715 (2015).

27. S. Halstenberg, T. Heimburg, T. Hianik, U. Kaatze, R Krivanek, Biophys. J. 75, 264 (1998).

28. J. Griesbauer, S. Bössinger, A. Wixforth, M.F. Schneider, Phys. Rev. Lett. 108, 061909 (2012).

29. R.D. Keynes, D.J. Aidley, C.L.H. Huang, Nerve and Muscle (Cambridge University Press, New York, 2011).

30. C. Fillafer, M.F. Schneider, Protoplasma 253, 357 (2016).

31. D.A. Doyle, J. Morais Cabral, R.A. Pfuetzner, A. Kuo, J.M. Gulbis, S.L. Cohen, B.T. Chait, R. MacKinnon, Science 280, 69 (1998).

32. B. Fichtl, S. Shrivastava, M.F. Schneider, Sci. Rep. 6 22874 (2016)

33. H. Träuble, M. Teubner, P. Woolley, H. Eibl, Biophys. Chem. 4, 319 (1976).

34. H. Trauble, H. Eibl, Proc. Natl. Acad. Sci. U.S.A. 71, 214 (1974).

35. C.B. Casper, D. Verreault, E.M. Adams, W. Hua, H.C. Allen, J. Phys. Chem. B 120, 2043 (2016).

36. I. Tasaki, P.M. Byrne, Biopolymers 32, 1019 (1992).

37. A. Blicher, K. Wodzinska, M. Fidorra, M. Winterhalter, T. Heimburg, Biophys. J. 96, 4581 (2009).

38. K. Græsbøll, H. Sasse-Middelhoff, T. Heimburg, Biophys. J. 106, 2143 (2014).

39. H.M. Seeger, M.L. Gudmundsson, T. Heimburg, J. Phys. Chem. B 111, 13858 (2007).

40. L.K. Sydnes, H. Holmsen, Biochem. Pharmacol. 61, 817 (2001).

41. M.J. Curran, M.S. Brodwick, J. Gen. Physiol. 98, 771 (1991). 